\title{
Mechanistic Aspects of the Formation of Aldehydes and Nitriles in Photosensitized Reactions of Aldoxime Ethers.
}

\author{
H. J. Peter de Lijser*, Natalie Ann Rangel, Michelle A. Tetalman, and Chao-Kuan Tsai \\ Department of Chemistry \& Biochemistry, California State University, Fullerton, CA 92834-6866. \\ E-mail: pdelijser@fullerton.edu
}

\section{Supporting Information}

Information From Theoretical Studies on $O$-Benzyl Benzaldehyde Oxime (3) Radical Cation

Information From Theoretical Studies on $O$-t-Butyl Benzaldehyde Oxime (4) Radical Cation 
Table S1. Information From Theoretical Studies on O-Benzyl Benzaldehyde Oxime (4) Radical Cation

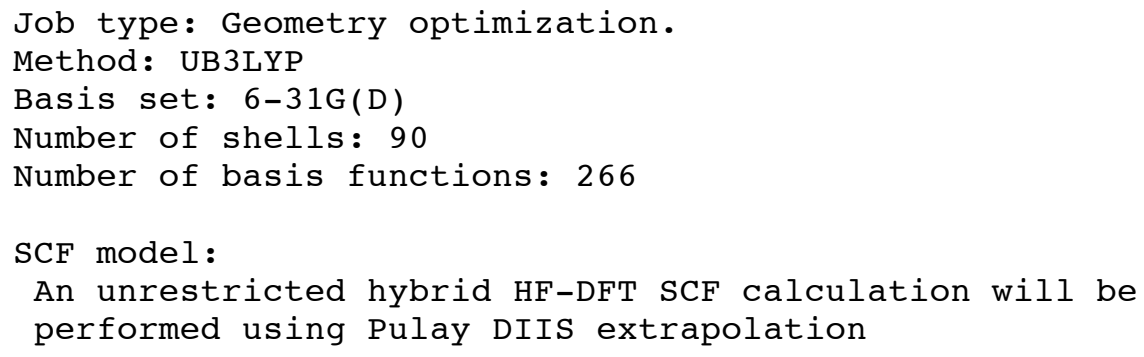

\begin{tabular}{|c|c|c|c|c|}
\hline & & Cartes & ian Coordinates & s (Angstroms) \\
\hline & Atom & $\mathrm{X}$ & $\mathrm{Y}$ & $\mathrm{Z}$ \\
\hline-- & -ーー-ーー & -ーーーーー-ーー-ーーー & ---- & -- \\
\hline $1 \mathrm{H}$ & $\mathrm{H} 1$ & -1.5355639 & .0047322 & -1.2128923 \\
\hline $2 \mathrm{C}$ & $\mathrm{C} 1$ & -1.6281993 & .0024749 & -.1257462 \\
\hline $3 \mathrm{~N}$ & $\mathrm{~N} 1$ & -.5300644 & .0027600 & .6189821 \\
\hline $4 \mathrm{C}$ & C9 & -2.9085564 & -.0001208 & .4874334 \\
\hline 50 & 01 & .5500872 & .0071571 & -.1528682 \\
\hline $6 \mathrm{C}$ & $\mathrm{C} 2$ & 1.8109286 & .0066487 & .6464433 \\
\hline $7 \mathrm{C}$ & C3 & 2.9550151 & .0030373 & -.3104297 \\
\hline $8 \mathrm{H}$ & $\mathrm{H} 4$ & 1.7626563 & -.8857216 & 1.2759578 \\
\hline $9 \mathrm{H}$ & H5 & 1.7654270 & .9015985 & 1.2724796 \\
\hline $10 \mathrm{C}$ & $\mathrm{C} 4$ & 5.0896272 & -.0040722 & -2.1202030 \\
\hline $11 \mathrm{C}$ & $\mathrm{C} 5$ & 3.4960292 & 1.2114918 & -.7664553 \\
\hline $12 \mathrm{C}$ & $\mathrm{C} 6$ & 3.4883392 & -1.2090626 & -.7663217 \\
\hline $13 \mathrm{C}$ & C7 & 4.5541263 & -1.2122270 & -1.6694285 \\
\hline $14 \mathrm{C}$ & $\mathrm{C} 8$ & 4.5618447 & 1.2076353 & -1.6695311 \\
\hline $15 \mathrm{H}$ & $\mathrm{H} 2$ & 3.0919542 & 2.1561418 & -.4107206 \\
\hline $16 \mathrm{H}$ & H6 & 3.0782606 & -2.1510312 & -.4103428 \\
\hline $17 \mathrm{H}$ & $\mathrm{H} 7$ & 4.9667846 & -2.1550647 & -2.0151343 \\
\hline $18 \mathrm{H}$ & H8 & 4.9805408 & 2.1477423 & -2.0154058 \\
\hline $19 \mathrm{H}$ & H9 & 5.9218034 & -.0067237 & -2.8178442 \\
\hline $20 \mathrm{C}$ & C10 & -5.4582118 & -.0042407 & 1.6210924 \\
\hline $21 \mathrm{C}$ & C11 & -3.0625828 & -.0024796 & 1.9092347 \\
\hline $22 \mathrm{C}$ & $\mathrm{C} 12$ & -4.0649954 & .0001696 & -.3467890 \\
\hline $23 \mathrm{C}$ & C13 & -5.3242294 & -.0018496 & .2188854 \\
\hline $24 \mathrm{C}$ & $\mathrm{C} 14$ & -4.3268901 & -.0045881 & 2.4604466 \\
\hline $25 \mathrm{H}$ & H3 & -2.1774296 & -.0025903 & 2.5351630 \\
\hline $26 \mathrm{H}$ & $\mathrm{H} 10$ & -3.9433755 & .0020125 & -1.4261546 \\
\hline $27 \mathrm{H}$ & H 11 & -6.2085396 & -.0016426 & -.4096448 \\
\hline $28 \mathrm{H}$ & H 12 & -4.4537929 & -.0063693 & 3.5379994 \\
\hline $29 \mathrm{H}$ & H 13 & -6.4509933 & -.0058180 & 2.0617944 \\
\hline
\end{tabular}


Table s1. Information From Theoretical Studies on O-t-Butyl Benzaldehyde Oxime (3) Radical Cation

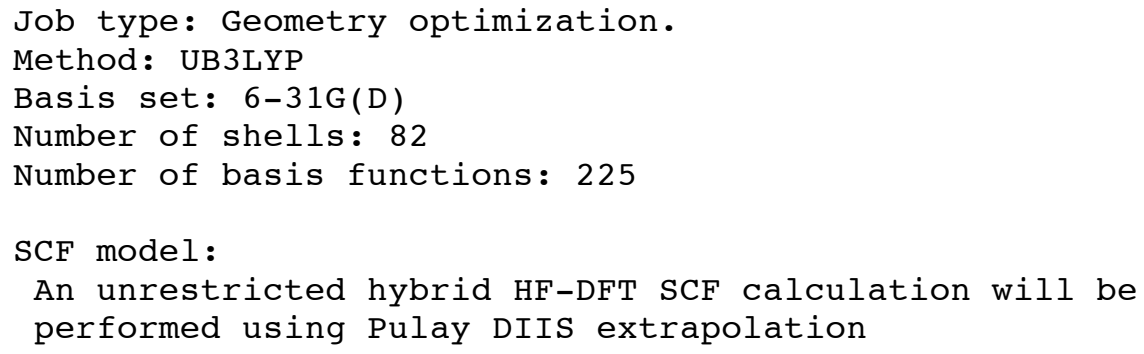

\begin{tabular}{rllrrr} 
& & & \multicolumn{2}{c}{ Cartesian Coordinates } & \multicolumn{1}{r}{ (Angstroms) } \\
1 & H & H 1 & \multicolumn{1}{c}{ X } & \multicolumn{1}{c}{ Y } & \multicolumn{1}{c}{ - } \\
2 & C & C1 & -0.6081125 & -2.0498869 & 0.0048155 \\
3 & N & N1 & -0.7800912 & -0.9725140 & 0.0022513 \\
4 & C & C6 & 0.2676538 & -0.1512075 & 0.0003668 \\
5 & O & O1 & -2.0991514 & -0.4556584 & 0.0009726 \\
6 & C & C2 & 1.3889936 & -0.8428505 & 0.0018775 \\
7 & C & C3 & 2.6571708 & 0.0050587 & 0.0001373 \\
8 & C & C5 & 3.7453430 & -1.0610983 & 0.0013740 \\
9 & C & C4 & 2.6450332 & 0.8447916 & 1.2748637 \\
10 & H & H2 & 2.6443619 & 0.8410586 & -1.2770421 \\
11 & H & H6 & 4.7234548 & -0.5709334 & 0.0004181 \\
12 & H & H7 & 3.6802569 & -1.6938153 & -0.8885522 \\
13 & H & H5 & 3.6807127 & -1.6912898 & 0.8931231 \\
14 & H & H8 & 3.5761675 & 1.4128770 & -1.3294643 \\
15 & H & H9 & 1.8101441 & 1.5476130 & -1.2860082 \\
16 & H & H4 & 2.5874495 & 0.2036959 & -2.1647991 \\
17 & H & H10 & 3.5768178 & 1.4168436 & 1.3250877 \\
18 & H & H11 & 2.5887619 & 0.2100142 & 2.1645210 \\
19 & C & C7 & 1.8107227 & 1.5512465 & 1.2823255 \\
20 & C & C8 & -4.7285052 & 0.4789612 & -0.0015311 \\
21 & C & C9 & -3.1897811 & -1.3761457 & 0.0029382 \\
22 & C & C10 & -2.3620473 & 0.9512018 & -0.0023049 \\
23 & C & C11 & -3.6646484 & 1.4030583 & -0.0034958 \\
24 & H & H3 & -4.4881512 & -0.9091723 & 0.0016752 \\
25 & H & H12 & -2.9858678 & -2.4429316 & 0.0054650 \\
26 & H & H13 & -1.5282214 & 1.6438895 & -0.0037352 \\
27 & H & H14 & -3.8747193 & 2.4675645 & -0.0059549 \\
28 & H & H15 & -5.3218152 & -1.6034357 & 0.0031597 \\
& & & -5.7519323 & 0.8430649 & -0.0024842
\end{tabular}

